\title{
Joint ZF and Partial ML Detection for Multi Cellular Network Environment
}

\author{
Won Seok Choi and Jeong Gon Kim ${ }^{1^{*}}$ \\ Dept. of Electronic Engineering \\ Korea Polytechnic University, Shi Heung City, Kyunggi Do, 429-793, Korea \\ *jgkim@kpu.ac.kr
}

\begin{abstract}
In this paper, we discuss joint detection schemes for an uplink cellular system when Base Station (BS) cooperation is possible for multi-cell users in a multi-cell scenario. Further, we analyze and evaluate the ML, ZF and ZF based SIC detection schemes. Although ML attains optimal performance, its complexity increases exponentially. Further, although ZF is simple, but exhibits the poor performance and SIC may give rise to error propagation. We propose a novel detection scheme, which combines the $Z F$ detection and partial ML decoding scheme in order to improve the detection performance as well as to decrease the decoder complexity. Simulation results show that the proposed scheme attains nearly $2 d B$ reduction in the required SNR values to achieve the same BER performance. Further, the proposed scheme can be applied to MIMO detection for a single user and extended to other types of multi-user and multi-antenna based future smart BS cooperation.
\end{abstract}

Keywords: MIMO detection, ML, ZF/MMSE, SIC, Base Station Cooperation

\section{Introduction}

The capacity of today's cellular mobile communication systems is mainly limited by inter-cell interference, which is the interference from neighboring cells. The solution to overcome this limitation, one possible option to mitigate this kind of interference is the multi-cell joint detection, which are based on the neighboring BS cooperation and it means that the neighboring signal across cell edge can be utilized rather than treating as interference. In general, it is widely accepted that future cellular systems can incorporate some form of multi-cell based signal processing, which can be a concept that requires the exchange of information among the involved base stations and joint detection based on its received signal and additional side information which are provided from the base station cooperation [1].

However, the multi-cell signal processing requires the additional backhaul rate that is required to exchange channel state or received signal information and the increased decoder complexity by implementing the centralized joint detector among the involved cooperating base stations compared with single detection schemes [2-5].

MIMO detection for single user and multiple antennas can be considered as the joint detection scheme for this situation if the involved base stations can perform the perfect cooperation. [6, 7] Traditional Maximum Likelihood (ML) detection can be used for optimal decoding, but the decoder complexity make its practical application be impossible. Therefore, low complexity receivers such as Zero-Forcing with Successive Interference Cancellation (ZF-SIC) was proposed (see $[8,9]$ ).

However, it is well known that the performance limitation of ZF based SIC scheme is due to the error propagation of the first decoded symbol to the next decoding symbol and

* Corresponding Author 
the decoder complexity due to the computation of inverse type of channel matrix in each step is still high as the number of antennas of transmitter and receiver is increased $[10,11]$.

In order to overcome this limitation, we propose the new detection scheme which can provide both the better BER performance and the reduced complexity compared with joint ZF based SIC detection scheme. The proposed scheme combines the linear ZF detection and the partial ML decoding concept to increase the reliability of the first decoded symbol, which can avoid the following error propagation effect to next step.

Simulation Results show that the proposed scheme attains the a little bit better BER performance and similar or reduced complexity over the ZF based SIC scheme in various scenarios of the involving mobile stations in uplink multiple cellular communication

In section II, the structure of simple uplink BS cooperation model is explained. In Section III, the brief description of ML, ZF and ZF based SIC MIMO detection schemes are provided. In Section IV, the joint $\mathrm{ZF}$ and partial ML detection scheme are proposed and analyzed. In Section V, BER performance of the proposed scheme are presented and the performance and complexity comparison and with other conventional approaches are also evaluated and then conclusions are made in Section VI.

\section{System Model}

We consider a uplink transmission from $M$ terminals (UEs) with one antenna each to $N$ base stations (BSs) with one antenna.

We can state the received $N$ vector $\mathbf{r}=\left[r_{1}, r_{2}, \cdots, r_{N}\right]^{T}$ is corresponded to the transmitted vector $\mathbf{s}=\left[s_{1}, s_{2}, \cdots, s_{M}\right]^{T}$ can be represented as

$$
\mathbf{r}=\mathbf{H} \mathbf{s}+\mathbf{n}
$$

where $\mathbf{r} \in \mathbf{C}^{[N \times]]}$ are the signals received at the BSs, $\mathbf{H} \in \mathbf{C}^{[N \times M]}$ is the channel matrix, $\mathbf{s} \in \mathbf{C}^{[M \times 1]}$ are the symbols transmitted from the UEs, and $\mathbf{n} \in \mathbf{C}^{[N \times 1]}$ is the complex gaussian noise vector with zero mean and unit variance.

The channel matrix $\mathbf{H}$ can be described as

$$
\mathbf{H}=\left[\begin{array}{cccc}
h_{11} & h_{12} & \cdots & h_{1 M} \\
h_{21} & h_{22} & \cdots & h_{2 M} \\
\vdots & \vdots & \ddots & \vdots \\
h_{N 1} & h_{N 2} & \cdots & h_{N M}
\end{array}\right]
$$

where $h_{i j}$ represents the channel gain from transmitted UE $j$ to received BS $i$.

Figure 1 represents the system under investigation in this paper, which means the 3-cell based scenario with 3 BS and 3 MS and the location of MS of each cell can be anywhere in his own cell and for simplicity, all of three MS and BSs have single antenna configuration. 


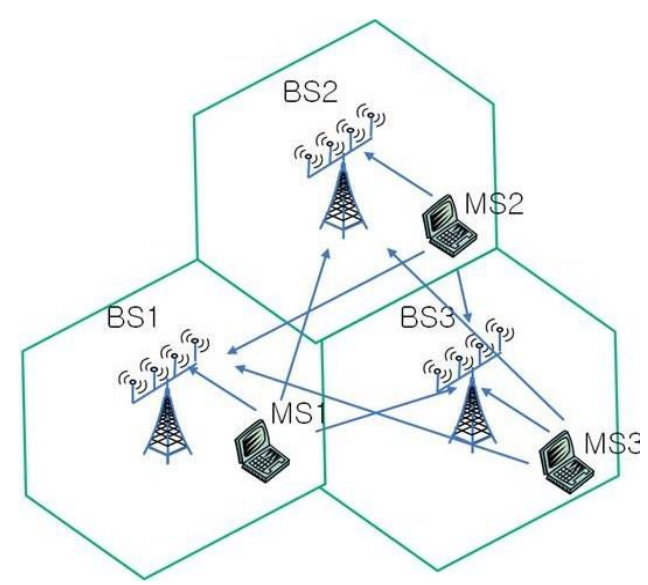

Figure 1. 3-cell Uplink Cellular System with 3 BS and 3 MS System

In order to enable the perfect Base Station Cooperation, the BSs are connected through an error-free backhaul link to central signal processing The transmission is disturbed by additive i.i.d. Gaussian noise, the channel assumed to be perfectly estimated and Rayleigh flat fading channel and the BSs and MSs are assumed to be fully synchronized in time and frequency.

\section{Detection Algorithms}

In this section, we review ML, the linear detection with $\mathrm{ZF}$ and the combination of nonlinear detection SIC with respect to ZF detection.

\subsection{Detection}

The optimum receiver is derived from the maximum likelihood criterion since the noise sequences at the antennas are assumed to be independent.

Thus the ML algorithm obtains, $\hat{\mathbf{s}}_{M L}$, the estimate of the transmit data vector $\mathrm{s}$ can be written as

$$
\hat{\mathbf{s}}_{M L}=\underset{\mathbf{s} \in \gamma^{M}}{\arg \min }\|\mathbf{r}-\mathbf{H} \mathbf{s}\|^{2}
$$

where $\gamma$ is the constellation size of data stream.

Even though the performance of the MLD is the best scheme among all detection algorithms, it is now impractical because of its high computation complexity $[12,13]$.

\subsection{ZF Algorithm}

One way to reduce the decoder computation complexity is the use of linear nulling approach, which is based on the ZF (zero forcing) criterion. The zero-forcing based matrix filter, $\mathbf{w}_{Z F}$, is given by the Moore-Penrose pseudo-inverse matrix, which is defined as $\mathbf{H}^{+},[5]$

$$
\mathbf{W}_{\mathrm{ZF}}=\mathbf{H}^{+}=\left(\mathbf{H}^{\mathrm{H}} \mathbf{H}\right)^{-1} \mathbf{H}^{\mathbf{H}}
$$

\subsection{ZF based SIC Detection}

$\mathrm{ZF}$ is a kind of linear nulling approaches and their performance can be boosted by 
being in conjunction with nonlinear techniques such as successive interference cancellation. In the symbol level interference cancellation, interference from already detected components of detected symbol vector $\hat{\mathbf{s}}$ is subtracted from the received signal vector $\mathbf{r}$, which results in a modified received vector in which, effectively, less interference is present.

SIC here indicates the multi stages interference cancellation. In each stage, one symbol is detected, regenerated and then the interference from it is cancelled from the received signal vector. This continues until all symbols transmitted at one time are detected.

In SIC, the signal detection is done in signal strength descending order. In [9], if we define $\mathbf{w}_{k_{i}}^{+}$denotes as the $k_{i}$ th row of the matrix filter $\mathbf{w}$ of the receiver, it shows that choosing the stronger signal strength is equivalent to choose smaller $\left\|\mathbf{w}_{k_{i}}^{+}\right\|$in each step. We give the algorithm process of ZF based SIC detection described below [10,11].

Step 1. Initialization

$$
\begin{aligned}
\mathbf{W}_{1} & =\mathbf{W}_{Z F} \\
i & =1
\end{aligned}
$$

Step 2. Equation represents the minimum row norm of matrix, namely, the $k_{i}$ th row

$$
k_{i}=\underset{j \notin\left\{k_{1}, \ldots k_{i-1}\right\}}{\arg \min }\left\|\left(\mathbf{W}_{i}\right)_{j}\right\|^{2}
$$

Step 3. Employing the weighted vector of $k_{i}$ th row reconstructed the transmitted signal of $k_{i}$ th layer and is listed below

$$
\begin{aligned}
& \mathbf{y}_{k_{i}}=(\mathbf{W})_{k_{i}} \mathbf{r}_{i} \\
& \hat{\mathbf{s}}_{k_{i}}=Q\left(\mathbf{y}_{k_{i}}\right)
\end{aligned}
$$

Step 4. Then we can eliminate the $k_{i}$ th signal from channel matrix and received using the following equations respectively

$$
\mathbf{r}_{i+1}=\mathbf{r}_{i}-\hat{\mathbf{s}}_{k_{i}}(\mathbf{H})_{k_{i}}
$$

Step 5. Delete $k_{i}$ th column of channel matrix $\mathbf{H}$ and by using this, find new receiver filter matrix $\mathbf{w}_{i+1}$ whch is replaced by $\mathbf{H}_{k_{i}}^{+}$,

$$
\begin{array}{r}
\mathbf{W}_{i+1}=\mathbf{H}_{k_{i}}^{+} \\
i=i+1
\end{array}
$$

Step 6. Repeat recursively the previous step until all transmitted symbols are detected

\section{Joint ZF and Partial ML Detection}

The ZF based SIC algorithms are still complex when there are many antennas in the system since it needs to compute the Moore-Penrose inverse matrix in every stage in order to calculate the updated weight filter matrix for decoding. 
In this paper, in order to reduce the decoder complexity and to increase the reliability of the first decoded symbol in successive decoding operation, we propose a new joint ZF and partial ML detection scheme, which calculate the inverse matrix only once and apply the partial ML decoding for other remaining symbols to be detected more reliably.

The details of the proposed scheme is depicted as follows.

Step 1. Initial Detection by ZF Decoding and determine $\overline{\mathrm{s}}$ as the reference signal for next partial ML decoding.

$$
\overline{\mathbf{s}}=\left(\mathbf{H}^{H} \mathbf{H}\right)^{-1} \mathbf{H}^{H} \times \mathbf{r}
$$

Step 2. Decide the order of decoding by descending order of the received SINR (Signal to Interference and Noise Ratio) of the received symbol vector $\mathbf{r}_{i}$.

$$
\operatorname{SIN} R_{\mathbf{r}_{i}}=\frac{\left\|h_{i i}\right\|^{2}}{\sigma_{n}{ }^{2}+\sum_{\substack{j=1 \\ j \neq i}}^{M}\left\|h_{i j}\right\|^{2}}
$$

where $\sigma_{n}{ }^{2}$ is the variance of the Gaussian noise vector.

Step 3. Apply the partial ML decoding only to the first symbol vector, which results from the determination of decoding order in step 2, when other symbol vectors except the first decoding symbol vector have the fixed value, which are already determined by initial ZF Decoding in Step 1. For example, if we assume the first decoding symbol is $\tilde{s}_{1}$, the remaining initial $\bar{s}$ vectors, $\overline{\mathbf{s}}_{2}, \overline{\mathbf{s}}_{3}, \cdots, \overline{\mathbf{s}}_{M}$, can be determined from the decoding results from Step 1 and then apply the partial ML decoding only for $\mathbf{s}_{1}$ in order to get, $\hat{s}_{1, M L}$, which will results the first decoded symbol vector from partially applied ML detection and this results can be utilized to next step for next iterative decoding operation.

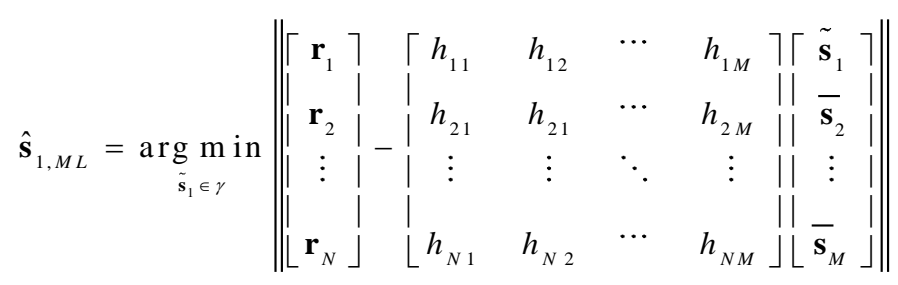

Step 4. Repeat partial ML Decoding operation in Step 3 in the same manner to other remaining symbol vector s according to the decoding order in Step 2 until all the values of symbol vector s are finally recovered

\section{Simulation Results}

In this section we present some numerical results and the comparisons of BER performances of proposed \& conventional detection schemes for various scenarios.

Table I represents some physical layer parameters of target system and most of them are in line with IEEE $802.16 \mathrm{~m}$ EMD documents and we produce the sample data based on these system parameters. As a noise and interference model, AWGN (Additive White 
Gaussian Noise), path loss due to the distance between BS and MS and Rayleigh flat multi-path fading are assumed in this paper.

Table 1. Physical Parameters of Target System $[15,16]$

\begin{tabular}{|c|c|}
\hline Parameter & Value \\
\hline Cell radius & $1500 \mathrm{~m}$ \\
\hline Number of Cell & 3 \\
\hline Number of MS & 3 \\
\hline Number of antenna & BS : $1, \mathrm{MS}: 1$ \\
\hline Number of data & 1024 \\
\hline FFT size & 1024 \\
\hline Modulation & QPSK \\
\hline Pathloss & $130.19+37.6 * \log 10(\mathrm{R} / 1000) \mathrm{dB}$ \\
\hline Sampling frequency & $11.2 \mathrm{MHz}$ \\
\hline Doppler frequency & $6.944 \mathrm{~Hz}$ \\
\hline Fading channel & Rayleigh channel \\
\hline
\end{tabular}

Figure 2 shows that the BS cooperation based multi-cell environment for the locations of BS and MS in the case of $M=3$ and $M=4$ in order to evaluate the detection performance, respectively.
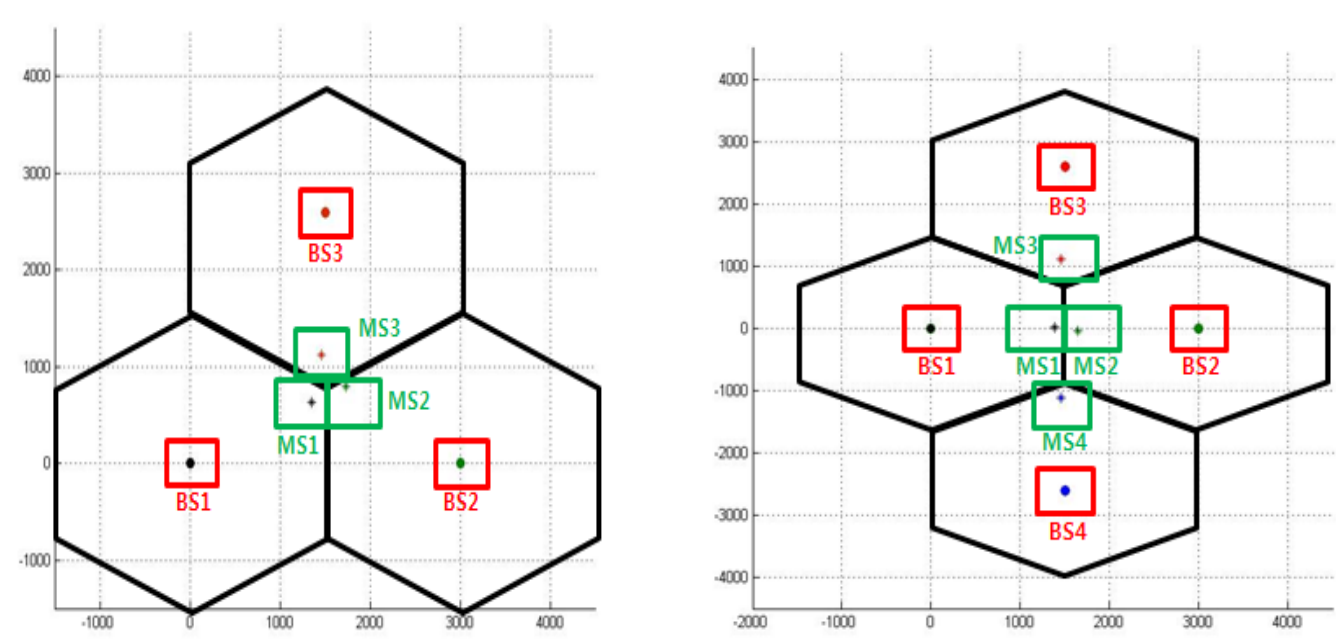

Figure 2. Cell Edge Scenario for the locations of BS and MS when the Number of Cooperating BS and MS is 3 and 4, Respectively

We assume Cell Edge scenario, which means that the distance between BS and MS is more than $80 \%$ of cell radius, hence, this assumption corresponds to nearly one of the worst case scenario for the locations of the involved BS and MS in multi-cellular communication.

Figure 3 and Figure 4 represent the BER performance comparison between proposed scheme and conventional detection algorithms. 


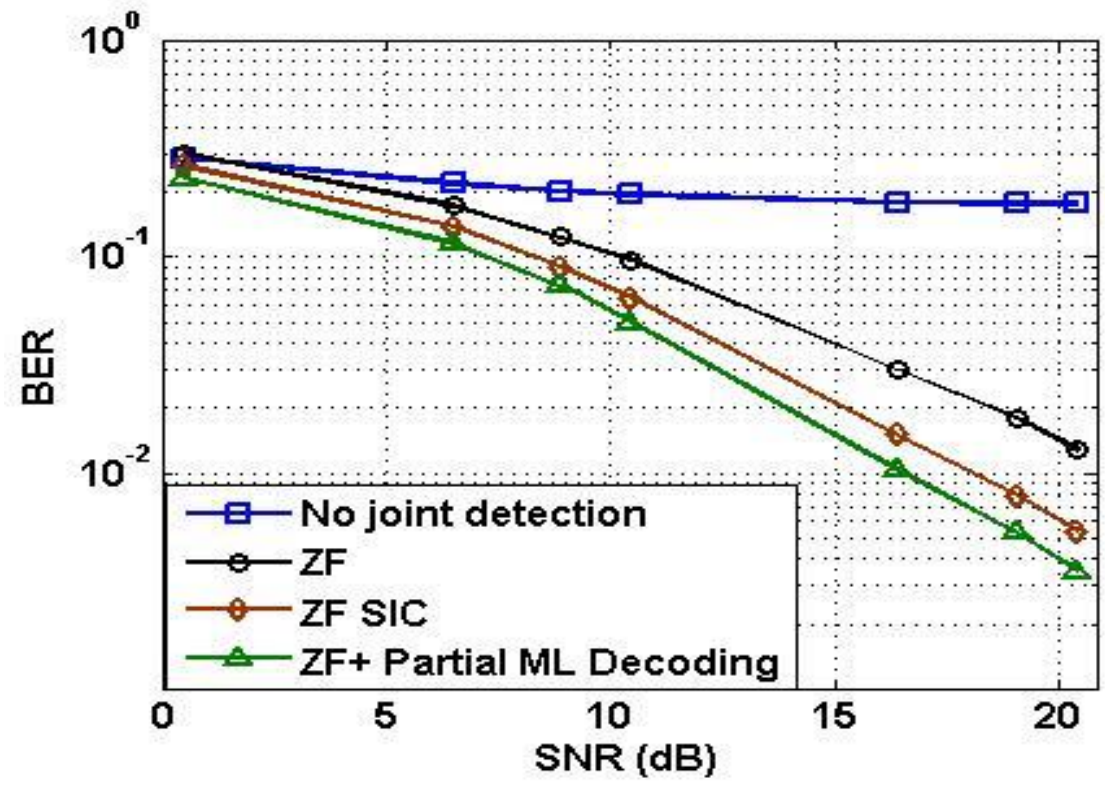

Figure 3. BER Performance Comparison of Joint Detection Algorithms when Three BSs are Cooperated

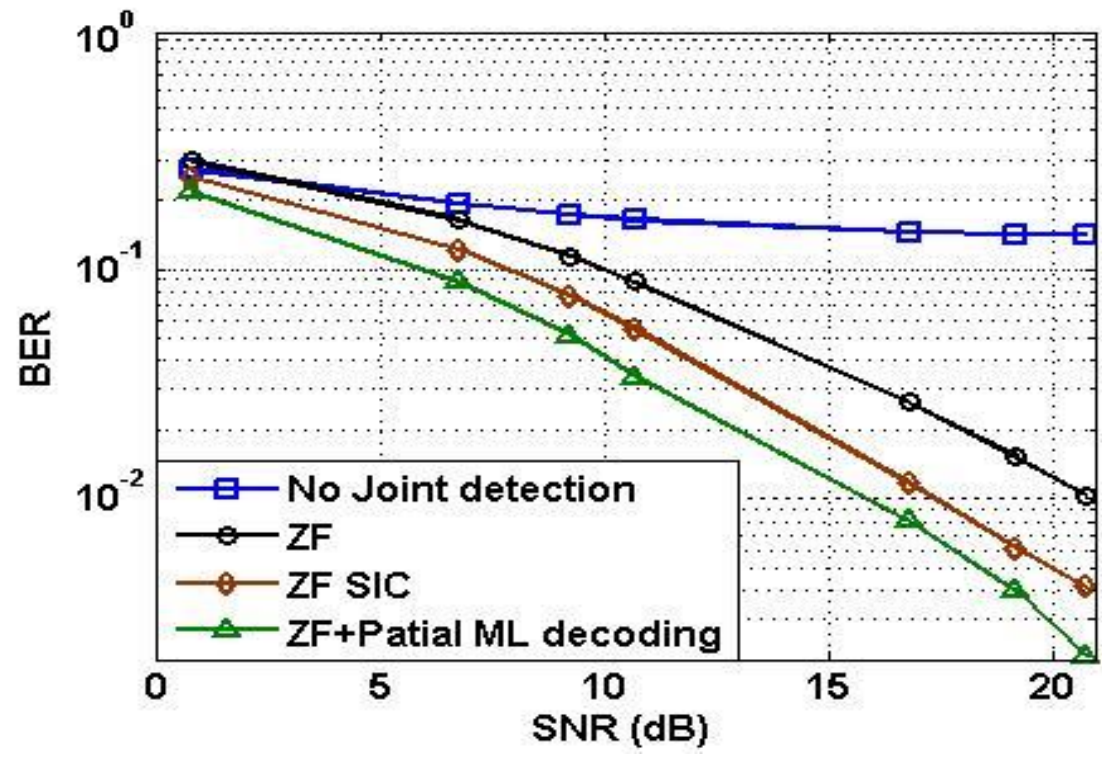

Figure 4. BER Performance Comparison of Joint Detection Algorithms when Four BSs are Cooperated

Figure 3 and Figure 4 compare the BER performances of ZF, ZF-SIC and proposed scheme for two different cases, which is the number of cooperative Base Station is 3 and 4 , respectively.

No joint Detection means that no cooperation is made between neighboring Base Stations. The proposed scheme attains nearly $4 \sim 5 \mathrm{~dB}$ reduced required SNR value over $\mathrm{ZF}$ and $1.5 \sim 2 \mathrm{~dB}$ reduced required SNR value over ZF-SIC scheme in order to achieve the same BER performance.

These figures show that the Base Station Cooperation seems to be imperative to guarantee the minimum detection performance in some range of cell border. 


\section{Conclusion}

In this paper, we investigated the uplink joint detection techniques for uplink cellular system when base station cooperation is possible in multi-cell scenario.

Traditional approaches for MIMO detection, such as ML, ZF and ZF based SIC detection, are considered as the joint detection for multi-cell based uplink mixed received signals from the Base Station Cooperation.

A new joint $\mathrm{ZF}$ and partial ML detection scheme was proposed to reduce the decoder complexity and also to improve the detection performance of multi-cell based multiple received signals. Simulation results show that the proposed scheme attains the improved BER performance over ZF and ZF based SIC scheme and also additionally achieves much reduced decoder complexity as the number of BS cooperation is increased since it does not need to compute inverse matrix operation in each stage.

The proposed scheme can be also applied to the single user MIMO detection and effectively applied as the number of antenna or cooperating Base Station are increased in multi-user and multi-antenna based BS Cooperation.

In addition, joint detection in cell border can have an advantage of detection performance if we use the uplink Base Station Cooperation between neighboring cell site. However, this requires additional backhaul link communication of the received signals and channel state information, hence, the limitation backhaul network capacity can be one of the important issue to be implemented in real system.

Backhaul link load reduction can be achieved through the reduction of the channel state information and the transfer of quantized version of received signal. Moreover, they can be combined with the joint detector with distributed type of decoding to reduce the complexity, hence, the simple and effective type of distributed approach with some backhaul link limitation should be investigated as a further works.

\section{Acknowledgement}

This research was supported by the MSIP (Ministry of Science, ICT and Future Planning), Korea, under the C-ITRC (Convergence Information Technology Research Center) support program (NIPA-2014-H0401-14-1003) supervised by the NIPA (National IT Industry Promotion Agency).

This paper is a revised and expanded version of a paper entitled "Combined Detection of ZF and Partial ML for Uplink Cellular BS Cooperation" presented at International Conference on Next Generation Computer and Information Technology 2014 (NGCIT 2014), October 24th 2014 at Liberty Central Saigon Hotel, Hochimin, Vietnam."

\section{References}

[1] P. Marsch, G. Fettweis , On Uplink Network MIMO under a Constrained Backhaul and Imperfect Channel Knowledge", IEEE International Conference on Communication (ICC) (2009).

[2] W. Hardjawana, B. Vucetic, L. Yonghui, "Multi-User Cooperative Base Station Systems with Joint Precoding and Beamforming", IEEE Journal Of Selected Topics In Singnal Processing, Vol. 3, No. 6, pp. 1079-1093, Dec. (2009).

[3] K. Balachandran, K. J.H., K. Karakayali, K. M. Rege, "NICE: A Network Interference Cancellation Engine for Opportunistic Uplink Cooperation in Wireless Networks", IEEE Transactions On Wireless Communications, Vol. 10, No. 2, pp. 540-549, Feb. (2011).

[4] V. Jagan Naveen, K. Murali Krishna, K. Raja Rajeswari, Performance Analysis of Equalization Techniques for MIMO Systems in Wireless Communication, International Journal of Smart Home, Vol. 4, No.4, pp. 47-63, October (2010).

[5] G. Dartmann, W. Afzal, G. Xitao, G. Ascheid, "Low complexity cooperative downlink beamforming in multiuser multicell networks", 12th IEEE International Conference on Communication Technology (ICCT), pp. 717-721, (2010) November 
[6] P. Srinivasa Rao, P. Dhana Raj, P. Asa Jyothi, S. Prasanna Lakshmi and S. SriLatha, Performance Analysis of MIMO Systems using TCM and Comparison with OSTBC, International Journal of Future Generation Communication and Networking, Vol. 6, No.4, pp. 99-110, August (2013).

[7] A. Goldsmith, S. A. Jafar, N. Jindal, and S. Vishwanath, "Capacity Limits of MIMO Channels, IEEE Journal on Selected Areas in Communications", Vol. 21, No. 5, pp. 684-702, June (2003).

[8] D. Tse and P. Viswanath, Fundamentals of Wireless Communication, Cambridge University Press, (2005).

[9] G. Wang, D. Wang and D. Li, "An Efficient ZF-SIC Detection Algorithm in MIMO CDMA System", The 14th IEEE 2003 PIMRC, Vol. 1, pp. 1708-1711, (2003).

[10] S. Cai, Z. Duan and J. Gao, "Comparison of Different Virtual MIMO Detection Schemes for 3GPP LTE”, IEEE 5th WiCom 2009, , pp. 1-5, (2009) September.

[11] K. Lee, D. Cha and K. Lee, Iterative Interference Reduction with MMSE-FDE in the Downlink for a PB/MC-CDMA System, International Journal of Smart Home, Vol. 7, No.2, pp. 121 - 130, March (2013).

[12] Krishnan, G.K., Reddy, V.U, "High performance low complexity receiver for v-blast", IEEE SPAWC 2007, pp.1-5, (2007) June.

[13] W. Xu, Y. Wang, Z. Zhou and J. Wang, "A computationally efficient exact ML sphere decoder", IEEE GLOBECOM 2004, pp. 2594-2598, (2004) December.

[14] P. W. Wolniansky, G.J. Foschini, G.D. Golden and R.A. Valenzuela, "V-BLAST: an architecture for realizing very high data rates over the rich-scattering wireless channel", 1998 URSI International Symposium on Signals, Systems, and Electronics, pp. 295-300, (1998) October

[15] Md. Didarul Alam and Md. Rezaul Huque Khan, Comparative Study of Path Loss Models of WiMAX at $2.5 \mathrm{GHz}$ Frequency Band, International Journal of Future Generation Communication and Networking, Vol. 6, No.2, pp. 11-24, April (2013).

[16] IEEE 802.16m Standardization Group, "802.16m Evaluation Methodology Document", v. 1.0, Jan. (2009)

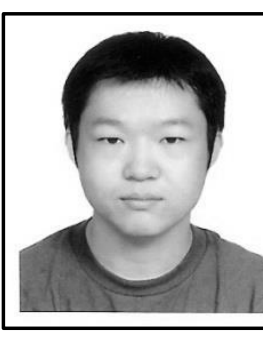

Won Seok Choi, was born in Seoul, Korea on Feb. 18, 1985. He received his B.S and M. S. degrees in Electronic Engineering at Korea Polytechnic University, Korea, in 2009 and 2012, respectively. His recent research interests are the interference cancellation for WBAN system and design of physical layer of $5 \mathrm{G}$ mobile cellular communication, specially MIMO, ICS repeater, femto cell network and cooperative communication.

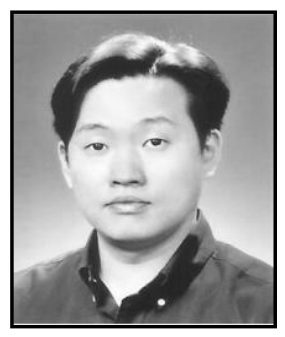

Jeong Gon Kim (Corresponding author), Jeong Gon Kim was born in Seoul, Korea on May 24, 1969. He received the B.S., M.S. and Ph.D, degrees all in electrical engineering from Korea Advanced Institute of Science and Technology (KAIST), Daejeon, Korea in 1991, 1993 and 1998, respectively. From 1998 to 1999, he was the Post Doctoral Research Fellow at the University of Hawaii at Manoa, USA. From 1999-2001, he joined R\&D center of LG Telecom, Korea and is involved in IMT-2000 radio access technology development.

From 2001-2003, he was also involved in 3GPP physical layer standardization, concentrating on the TDD mode in the Telecommunication Research center of Samsung Electronics.

Since 2003, he is now a Professor at the Department of Electronic Engineering of Korea Polytechnic University.

His research interests now include the design and performance analysis of wireless communication system, specially $5 \mathrm{G}$ radio access network, error control coding techniques and short range wireless communication system.

(Tel: +82-31-8041-0486, Fax: +82-31-8041-0499, E-mail: jgkim@kpu.ac.kr) 
International Journal of Control and Automation Vol. 7, No. 11 (2014) 